\title{
propagation de fractures dans des roches sous sollicitations thermo-hydro-mécaniques
}

\author{
propagation of fractures in rocks subjected \\ to thermo-hydro-mechanical stresses
}

\author{
G. BERTHOMIEU - P. JOUANNA \\ Laboratoire de Génie Civil, \\ Université des Sciences et Techniques du Languedoc (")
}

Rev. Franç. Géotech n 47 , p.p. 5-16 (avril 1989)

\section{Résumé}

L'approche proposée pour l'étude de la propagation dans des roches, sous sollicitations thermo-hydro-mécaniques complexes, consiste à recréer en laboratoire des conditions aussi proches que possible de celles rencontrées sur le site. Cette mise en situation des champs de températures et de contraintes peut être envisagée soit globalement sur un domaine de roche suffisamment important pour représenter la réalité in situ, soit uniquement sur un sous-domaine, au voisinage de l'extrémité de fissure. On s'attachera essentiellement à la deuxième approche, seule praticable dans l'exemple emprunté au cadre historique de la géothermie en roches chaudes sèches.

Après avoir rappelé les fondements théoriques de la thermo-mécanique de la rupture, on donnera la banque de résultats actuellement disponible dans des expériences de propagation de fractures, en situations thermo-hydro-mécaniques complexes. Enfin on passera en revue les difficultés actuelles pour prévoir, à partir des expériences de laboratoire, les conditions de rupture in situ.

\footnotetext{
Abstract

The approach proposed for the investigation of fracture propagation in rock under complex thermo-hydro-mechanical stresses consists of reproducing site conditions as closely as possible in the laboratory. This set-up of fields of temperatures and stresses can be envisaged applied either globally to a rock zone large enough to represent in situ reality or only to a sub-zone in the neighbourhood of the tip of the fracture. The investigation centred mainly on the second approach, which was the only method usable in the example taken within the framework of the geothermy of hot dry rock.

A summary of the theoretical basis of thermo-mechanics of failure is followed by the results available today drawn from experiments on fracture propagation in complex thermo-hydro-mechanical situations. The communication ends with examination of current difficulties to be expected in situ failure conditions on the basis of laboratory experiments.
} 


\section{INTRODUCTION}

\subsection{Cadre général de l'étude}

Cette étude a pour cadre la fracturation de roches sous l'action de sollicitations thermo-hydro-mécaniques complexes. Ce problème mal connu se rencontre dans des domaines importants tels que:

- les exploitations pétrolières où la fracturation thermique peut interférer avec la fracturation hydraulique lors de la stimulation des gisements ;

- le stockage de déchets radioactifs où la variation locale de température risque de créer de nouvelles fractures et perturber l'équilibre hydrodynamique souterrain :

- la géothermie profonde où l'injection dans des fractures d'un fluide plus froid que la roche est susceptible de modifier la perméabilité du massif ;

- le stockage de gaz liquéfiés

- le stockage de chaleur dans des roches, etc.

\subsection{Méthodes d'approche possibles}

Compte tenu de la complexité du problème, où interfèrent des processus physico-chimiques, thermiques, hydrauliques et mécaniques, la méthode d'investigation proposée consiste à mettre en situation, au laboratoire, la roche dans des conditions aussi proches que possible de celles rencontrées sur le site. Cette mise en situation peut s'envisager :

- soit directement, en identifiant les paramètres du site et du laboratoire, par une approche globale, de type système ou "boite noire ":

- soit indirectement, en identifiant d'abord des champs de variables locales avec retour ensuite aux paramètres systémiques, seuls accessibles en pratique à la mesure.

\subsubsection{Approche par mise en situation globale}

Une situation donnée, sur le site ou au laboratoire, peut être caractérisée globalement par des paramètres tels que : géométrie du domaine matériel, conditions initiales et chemins de sollicitations appliqués aux limites du domaine.

Cette méthode directe est rarement envisagée a priori en mécanique traditionnelle, domaine où l'on maîtrise bien les phénomènes élémentaires. Mais elle prend de plus en plus d'importance dans les domaines où de nombreux phénomènes sont couplés ou dans le cas de matériaux complexes.

L'avantage essentiel de cette approche est d'aller droit à la réponse, sans passer par l'intermédiaire d'hypothèses de comportement aussi nombreuses qu'incertaines. L'étude par exemple de la tenue de parois de forages, dans des roches hétérogènes et sous des cheminements thermodynamiques complexes, peut parfaitement bien s'envisager par cette mise en situation globale.

Mais la difficulté essentielle de cette approche tient à l'importance du domaine matériel à simuler au laboratoire. Par exemple, il devient impossible de simuler une fissure décamétrique, plongée dans un milieu infini.

\subsubsection{Approche par mise en situation locale}

Devant une telle difficulté, on pourra tenter de conserver l'idée de mise en situation, mais de façon locale. Ainsi dans le cas d'une fracture, sa propagation éventuelle est suspendue aux conditions rencontrées localement à son extrémité, seul sous-domaine matériel qu'il est suffisant de mettre en situation.

Les paramètres au laboratoire et sur le site n'ont plus rien apparemment de commun. On parle cependant d'équivalence entre ces paramètres, dès lors qu'ils engendrent les mêmes champs de variables locales, dans le sous-domaine matériel examiné.

Tout le problème de la mise en situation locale consiste à savoir dans quelle mesure on peut jouer sur les paramètres en laboratoire, afin de réaliser aussi rigoureusement que possible une identité entre les champs locaux sur le site et au laboratoire, sur le sous-domaine souhaité. En général il n'est pas possible de parvenir rigoureusement à cette identité et l'on devra consentir des approximations.

\subsection{Application proposée et contenu de l'étude}

\subsubsection{Application proposée}

On propose, pour illustrer cette étude, le cadre de la géothermie profonde en roches chaudes sèches. Dans cette application, pour récupérer la chaleur de roches situées à grande profondeur, on envisage de faire circuler de l'eau entre deux ou plusieurs forages, dans un réseau de fractures, créées ou réouvertes le plus souvent par fracturation hydraulique. Dans ces fractures qui jouent le rôle d'échangeur, la circulation d'un fluide plus froid que la roche peut favoriser, entre autres phénomènes, l'apparition en parois de nouvelles fissures, ou la propagation des fractures elles-mêmes.

La stabilité des parois de fracture a fait l'objet de précédentes publications [3, $4,6,8]$.

Nous nous limiterons, dans cet article, à l'effet de sollicitations thermiques sur la stabilité d'une extrémité de fracture, de grande extension, préalablement sollicitée hydrauliquement et mécaniquement. Cette application conduit à faire le choix d'une approche par mise en situation locale.

\subsubsection{Contenu de l'étude}

Sont donnés tout d'abord en 2. les fondements théo. riques de lapproche par mise en situation locale, dans le cas d'une fracture de grande extension sur le site :

- le paragraphe 2.1. donne le bilan des paramètres $\left(P_{5}\right)$ in situ et des paramètres $\left(P_{e}\right)$ envisagés au laboratoire :

- le paragraphe 2.2. aborde la détermination des champs de variables locales, sur le site ou au laboratoire, par l'intermédiaire de la thermo-mécanique de la rupture

- le paragraphe 2.3. conduit, sous les réserves de la thermo-mécanique de la rupture, aux relations d'équivalence entre paramètres au laboratoire et sur le site. 
Puis sont décrites en 3 . des expériences de rupture thermo-hydro-mécaniques en laboratoire ainsi qu'une première interprétation des résultats d'essais sur granite et calcaire ;

- le paragraphe 3.1. décrit le banc d'essais et le mode opératoire permettant de déterminer les paramètres à la rupture d'éprouvettes préfissurées ;

- le paragraphe 3.2. présente les résultats d'essais de rupture en situation thermo-hydro-mécanique complexe et les tentatives d'interprétation des résultats.

\section{FONDEMENTS THÉORIQUES}

\subsection{Paramètres in situ $\left(\mathrm{P}_{\mathrm{s}}\right)$} et au laboratoire $\left(\mathrm{P}_{\mathrm{e}}\right)$

\subsubsection{Paramètres in situ $\left(P_{s}\right)$}

Etude de cas in situ

La réalité de la fracturation rencontrée dans des échangeurs de géothermie profonde est souvent très complexe. Pour l'exposé de la méthode, on adoptera l'hypothèse d'école historique qui consiste à envisager que la fracture, perpendiculaire à la contrainte principale mineure, a la forme d'un ellipsoïde de révolution très aplati. Toutefois, cette simplification didactique n'est pas une limitation à la méthode générale, dans des cas plus complexes.

Paramètres in situ $\left(\mathrm{P}_{\mathrm{s}}\right)$

a. Géométrie (fig. 1)

La fracture idéalisée sur le site est ici supposée dans un plan vertical ; elle est caractérisée par un seul paramètre, son rayon $\mathrm{a}_{5}$.

b. Coefficients physiques et thermo-hydro-mécaniques de la roche

$\rho$ : masse volumique :

$\mathrm{k}$ : conductivité thermique, $\mathrm{c}$ : chaleur massique,

$\chi=\mathrm{k} / \rho \mathrm{c}=$ diffusivité thermique ;

$\phi$ : porosité, $\mathrm{K}$ : coefficient de perméabilité de Darcy

$\lambda, \mu$ : coefficients de Lamé (roche isotrope), $\alpha$ : coefficient de dilatation linéaire.

c. Conditions initiales thermo-hydro-mécaniques

- L'état de contraintes principales totales au loin est caractérisé par $\sigma_{1 s}, \sigma_{2 s}$ et $\sigma_{3 s}$.

- La fracture est baignée par de l'eau à une pression $\mathrm{p}_{\mathrm{fs}}$.

- La température du fluide $\mathrm{T}_{\mathrm{fs}}$ est égale à la température uniforme de la roche $T_{r s}$.

d. Paramètres de chargement thermo-hydro-mécaniques

- L'état de contraintes principales au loin est supposé constant.

- La pression $\mathrm{p}_{\mathrm{is}}$ de l'eau en fracture est supposée constante.

- La température de la roche au loin est supposée constante. Seule la température du fluide $T_{f s}$ variera et sera à considérer comme le paramètre de chargement du problème. Dans la suite cette température sera supposée représentative de la température du fluide, au voisinage de l'extrémité de fracture. En fait pour donner une définition exacte à cette variable de contrôle, il conviendrait d'effectuer une modélisation complète de toute la boucle thermique et de définir cette quantité en un point bien précis du système.

La loi d'évolution de cette variable de contrôle $T_{\text {fs }}$ est a priori quelconque. Dans l'analyse qui suit, on a supposé qu'il s'agissait d'un refroidissement monotone, simulé par la loi suivante, à trois paramètres $\Delta T_{s}, \tau_{s}$ et $t_{s}$ :

$T_{i s}=T_{r s}-\Delta T_{5} \cdot$ th $\left(t_{s} / \tau_{5}\right)$

avec :

$\Delta \mathrm{T}_{\mathrm{s}}=$ amplitude maximale du refroidissement ;

$\tau_{\mathrm{s}}=$ temps de relaxation $\left(\tau_{\mathrm{s}}=0\right.$ : choc thermique ;

$\tau_{\mathrm{s}}=\infty$ : régime permanent) ;

$\mathrm{t}_{\mathrm{s}}=$ temps de sollicitation (avec th $=$ tangente hyperbolique).

\subsubsection{Paramètres $\left(P_{e}\right)$ sur éprouvette en laboratoire}

\section{Etude de cas au laboratoire}

La conception du dispositif expérimental doit mettre en jeu un nombre de paramètres suffisant pour être à même de simuler, dans le sous-domaine matériel en fond de fissure, les champs thermo-hydromécaniques rencontrés in situ.

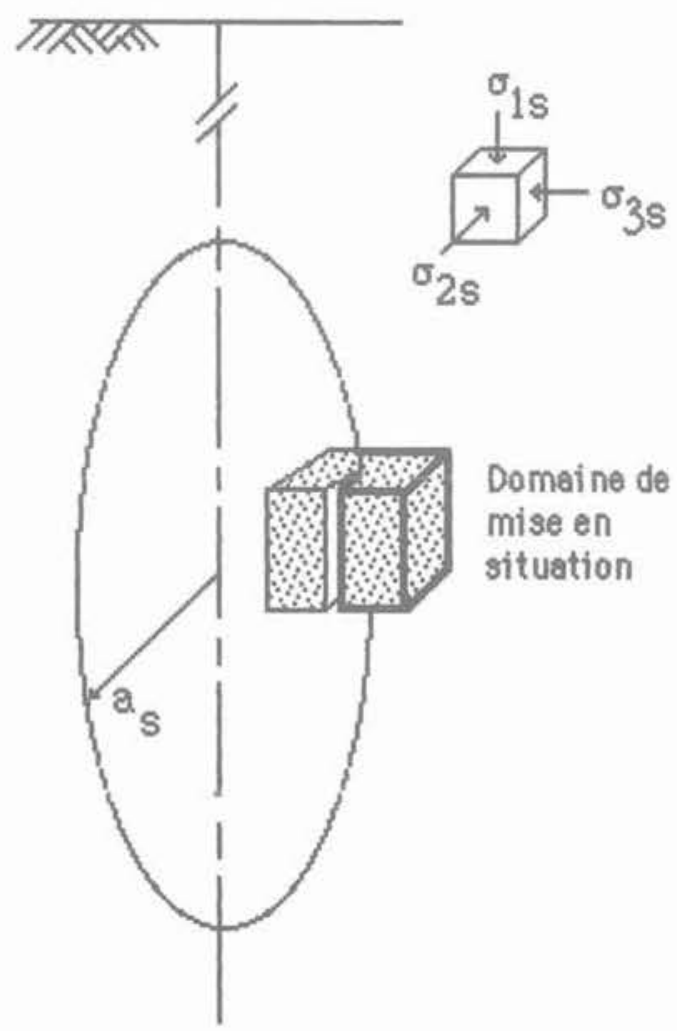

Fig. 1. - Fracture "idéalisée " sur le site. Fig. 1. - "ldealized "site fracture. 
Le dispositif adopté porte sur des éprouvettes de roche cylindriques, munies d'un forage central avec deux fissures longitudinales, dans lequel peut circuler de l'eau à pression et température variables. Le tout est plongé dans une étuve, et soumis à un état de contrainte biaxial de révolution autour de l'axe de l'éprouvette.

\section{Paramètres au laboratoire $\left(\mathrm{P}_{\mathrm{e}}\right)$}

a. Géométrie (fig. 2)

- Le rayon extérieur des éprouvettes est égal à $\mathrm{R}_{\mathrm{e}}$. - Le diamètre du forage intérieur est de $2 \mathrm{r}_{e}$.

- Les deux fissures longitudinales centrales, suivant deux directions opposées pour conserver la symétrie, ont une profondeur $\mathrm{a}_{\mathrm{e}}$.

- La hauteur de l'échantillon est égale à $\mathrm{H}_{\mathrm{e}}$.

b. Coefficients physiques et thermo-hydro-mécaniques de la roche

Ces coefficients sont supposés identiques in situ et sur l'éprouvette de laboratoire.

c. Conditions initiales thermo-hydro-mécaniques

- Etreinte latérale extérieure $p_{o e}$ et contrainte verticale $\sigma_{\text {ve }}$.

- L'eau située dans le forage baigne les lèvres de la fracture sous une pression $\mathrm{p}_{\mathrm{ie}}$.

- La température du fluide $\mathrm{T}_{\mathrm{fe}}$ est égale à la température initiale de la roche $\mathrm{T}_{\mathrm{re}}$.

\section{d. Paramètres de chargement thermo-hydro. mécaniques}

- L'état de contraintes extérieur ( $p_{o e}, \sigma_{\text {ve }}$ ) est supposé constant.

- La pression p pe de l'eau en fracture est supposée constante, sauf mention contraire.

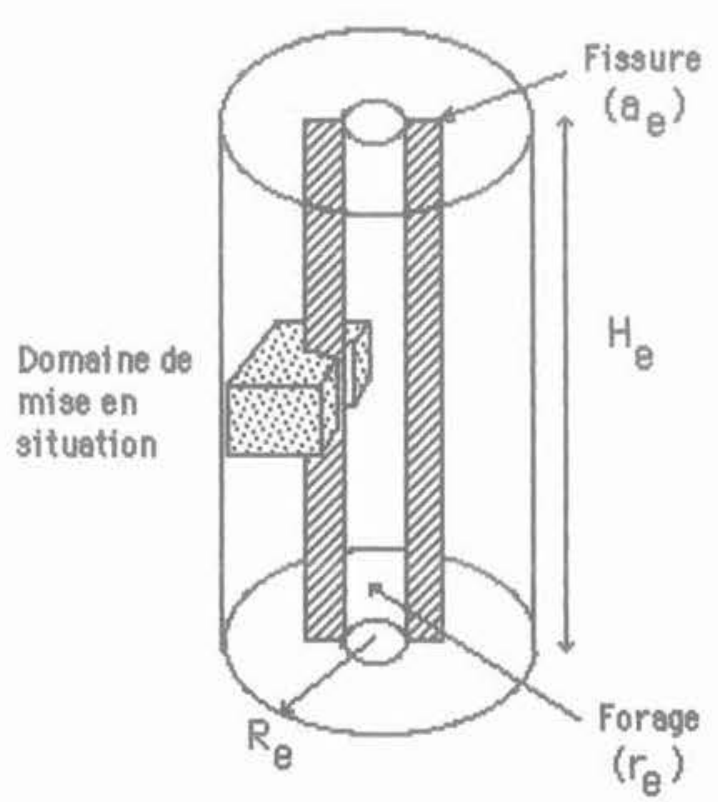

Fig. 2. - Eprouvette de laboratoire.

Fig. 2. - Laboratory sample.
- La température de l'étuve $\mathrm{T}_{\text {re }}$ est gardée constante et seule la température du fluide $T_{f e}$ dans le forage central variera et sera à considérer comme le paramètre de chargement du problème. Dans la suite cette température sera supposée représentative de la température du fluide au voisinage de l'extrémité de fracture. On verra les difficultés inhérentes à la mesure de cette variable de contrôle, capitale dans ce problème.

La loi d'évolution de cette variable de contrôle $T_{f e}$ est similaire à la loi de refroidissement adoptée sur le site, soit :

$\mathrm{T}_{\mathrm{fe}}=\mathrm{T}_{\mathrm{re}}-\Delta \mathrm{t}_{\mathrm{e}} \cdot \mathrm{th}\left(\mathrm{t}_{\mathrm{e}} / \tau_{\mathrm{e}}\right)$

avec :

$\Delta T_{e}=$ amplitude maximale du refroidissement ;

$\tau_{e}=$ temps de relaxation $\left(\tau_{e}=0\right.$ : choc thermique ;

$\tau_{e}=\infty$ : régirne permanent) :

$\mathrm{t}_{e}=$ temps de sollicitation (avec th = tangente hyperbolique)

2.2. Champs de variables locales, au voisinage de l'extrémité de fracture

\subsubsection{Caractérisation des champs de variables locales}

Problème général

Les champs de variables locales thermo-hydromécaniques, au voisinage de la fracture, portent sur : - les températures $\mathrm{T}(\mathrm{M}, \mathrm{t})$;

- les pressions d'eau $\mathrm{u}(\mathrm{M}, \mathrm{t})$;

- les tenseurs contraintes $\sigma(\mathrm{M}, \mathrm{t})$.

et la mise en situation rigoureuse voudrait que l'on ait identité sur le site et au laboratoire, entre ces trois champs, pour tout point $\mathrm{M}$ et tout temps $\mathrm{t}$, au voisinage de la fracture.

\section{Critères}

La comparaison de ces champs entre eux oblige à faire le choix de critères. Ce problème général n'est relativement aisé que sous les hypothèses restrictives de la mécanique de la rupture, qui ne traite actuellement que du cas de roches élastiques, et surtout monophasiques. Cette restriction est de taille et, faute de mieux pour le moment, on constatera ses insuffisances dans l'interprétation des résultats expérimentaux.

Les critères adoptés, pour le cas d'une roche monophasique, sont les suivants :

- pour les champs de température, on se contentera de comparer les températures de surface, supposées identiques aux paramètres de chargement $T_{f s}$ ou $\mathrm{T}_{\text {fe }}$;

- pour les contraintes totales, leur distribution sera normée par les facteurs d'intensité de contrainte $\mathrm{K}_{\mathrm{is}}(\mathrm{t})$ ou $\mathrm{K}_{\mathrm{le}}(\mathrm{t})$; les facteurs d'intensité de contraintes $\mathrm{K}_{11}(\mathrm{t})$ et $\mathrm{K}_{\text {III }}(\mathrm{t})$ sont supposés négligeables dans le cas présent.

Les critères à adopter, dans le cas d'une roche biphasique, sont encore du domaine des conjectures. En conséquence, les coefficients hydrauliques de la roche ( $\phi$ et K) n'interviendront pas dans la suite. 
2.2.2. Calculs des facteurs d'intensité de contraintes $K_{I s}(t)$ et $K_{I e}(t)$ en fonction des paramètres $\left(P_{s}\right)$ et $\left(P_{e}\right)$ - Cas monophasique

Le facteur d'intensité de contrainte $\mathrm{K}_{\mathrm{l}}(\mathrm{t})$, que ce soit sur le site ou au laboratoire, peut se décomposer sous la forme:

$$
\mathrm{K}_{\mathrm{l}}(\mathrm{t})=\mathrm{K}_{\mathrm{l}}^{0}+\Delta \mathrm{K}_{\mathrm{l}}(\mathrm{t})
$$

avec :

$K_{l}^{0}=$ facteur relatif à l'état initial ;

$\Delta \mathrm{K}_{\mathrm{I}}(\mathrm{t})=$ accroissement du facteur sous sollicitations thermiques.

\section{Calcul de $K_{I}^{0}$ aux états initiaux}

A partir de solutions analytiques (ou semi-numériques) établies dans la littérature pour les configurations et les chargements les plus usuels [13 par exemple], il est facile de trouver, pour les configurations géométriques adoptées, les expressions de $\mathrm{K}_{\mathrm{j}}^{0}$, sur le site et au laboratoire.

\section{a. Expression de $K_{l}^{0}$ sur le site}

Le sous-ensemble $\left(\mathrm{P}_{\mathrm{s}}^{0}\right)=\left(\mathrm{a}_{5}, \mathrm{P}_{\mathrm{is}}, \sigma_{3 \mathrm{~s}}\right)$ des paramètres in situ conduit à :

$$
\mathrm{K}_{\mathrm{ls}}^{0}=\frac{2}{\sqrt{\Pi}} \sqrt{\mathrm{a}_{\mathrm{s}}}\left(\mathrm{p}_{\mathrm{is}}-\sigma_{3 \mathrm{~s}}\right)
$$

b. Expression de $K_{I}^{0}$ au laboratoire

L'éprouvette a été choisie de façon à pouvoir considérer avec une très bonne approximation que la fissure se trouvait dans un milieu infini et que l'hypothèse de déformation plane était raisonnable.

Dans ces conditions, le sous-ensemble $\left(\mathrm{P}_{e}^{0}\right)=\left(\mathrm{a}_{e}\right.$, $\left.\mathrm{r}_{\mathrm{e}}, \mathrm{p}_{\mathrm{fe}}, \mathrm{p}_{\mathrm{oe}}\right)$ des paramètres au laboratoire conduit à :

$$
K_{l e}^{0}=\sqrt{\Pi\left(r_{e}+a_{e}\right)} \cdot\left(p_{f e}-p_{o e}\right)
$$

Calcul de $\Delta K_{l}(t)$ sous sollicitations thermiques

La détermination de $\Delta \mathrm{K}_{\mathrm{t}}(\mathrm{t})$ nécessite des calculs numériques, dont on peut optimiser l'exploitation grâce à un recours préalable à l'analyse dimensionnelle.

(a) SITE

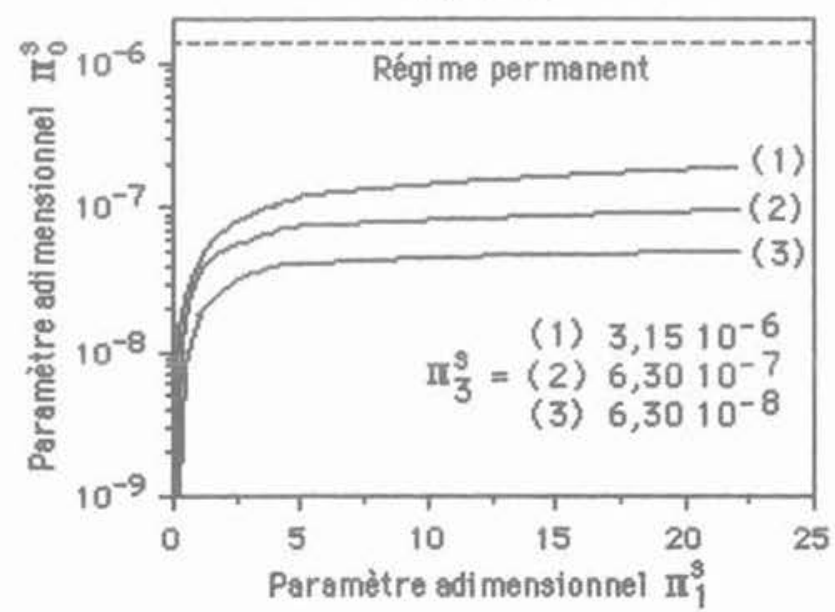

A. Contribution de l'analyse dimensionnelle [7, 8]

a. Sur le site

Le sous-ensemble $\left(\mathrm{P}_{\mathrm{s}}\right)$ des paramètres qui intervient ici est :

$\mathrm{P}_{\mathrm{s}}^{*}=\left\{\mathrm{a}_{\mathrm{s}}, \lambda_{\mathrm{s}}, \mu_{\mathrm{s}}, \alpha_{\mathrm{s}}, \Delta \mathrm{T}_{5}, \tau_{\mathrm{s}}, \chi_{\mathrm{s}}, \mathrm{t}_{\mathrm{s}}\right\}$

A l'aide du théorème de Vaschy-Buckingham lou théorème des II) et en ajoutant l'hypothèse de dilatation thermique linéaire, on peut regrouper ces paramètres sous forme de termes adimensionnels reliés entre eux par la relation :

$$
\frac{\Delta \mathrm{K}_{\mathrm{Is}}}{\sqrt{\mathrm{a}_{\mathrm{s}} \cdot \alpha_{\mathrm{s}} \cdot \Delta \mathrm{T}_{\mathrm{s}} \cdot \lambda_{\mathrm{s}}}}=g_{\mathrm{s}}\left(\frac{\mathrm{t}_{\mathrm{s}}}{\tau_{\mathrm{s}}}, \frac{\chi_{\mathrm{s}} \mathrm{t}_{\mathrm{s}}}{\mathrm{a}_{\mathrm{s}}^{2}}\right)
$$

encore notée :

$$
\Pi_{0}^{5}=\mathrm{g}_{\mathrm{s}}\left(\Pi_{1}^{\mathrm{s}}, \Pi_{2}^{\mathrm{s}}\right)
$$

b. Au laboratoire

Le sous-ensemble $\left(\mathrm{P}_{e}{ }^{*}\right)$ des paramètres qui intervient ici est :

$\mathrm{P}_{\mathrm{e}}^{*}=\left\{\mathrm{r}_{e}, \mathrm{R}_{\mathrm{e}}, \mathrm{a}_{e}, \lambda_{\mathrm{e}}, \mu_{e}, \alpha_{e}, \Delta \mathrm{T}_{e}, \tau_{e}, \chi_{e}, \mathrm{t}_{e}\right\}$

Pour des rapports $r_{e} / a_{e}$ et $R_{e} / a_{e}$ donnés, un calcul identique conduit à l'expression :

$$
\Pi_{0}^{e}=g_{e}\left(\Pi_{1}^{e}, \Pi_{2}^{e}\right)
$$

\section{B. Contribution du calcul numérique $[5,8]$}

La détermination des fonctions $g_{s}$ et $g_{e}$ nécessite une approche numérique. On a choisit la méthode des éléments finis et le code de calcul CASTEM, exploité en collaboration avec la CISI (Compagnie Internationale de Services en Informatique) et le CEA (Commissariat à l'Energie Atomique) de Cadarache, Les calculs sont faits en situation axisymétrique pour le site et en déformation plane pour le laboratoire. Le maillage de la fracture sur le site doit être très fin en raison de sa forme très aplatie.

Les résultats peuvent être représentés sous forme de graphes adimensionnels tels que ceux de la figure $3 a$ pour la fracture sur site et la figure $3 \mathrm{~b}$ pour la frac-

\section{(b) LABORATOIRE}

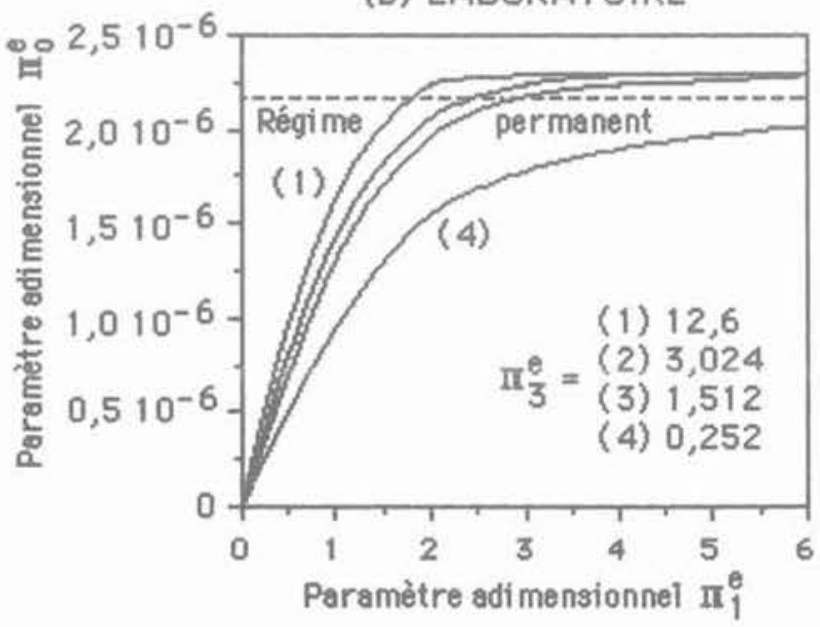

Fig. 3. - Graphes adimensionnels sur le site et au laboratoire. Fig. 3. - Adimensional graphs for site and laboratory. 
ture de l'éprouvette au laboratoire. Ils représentent la variation de $\Pi_{0}$ en fonction de $\Pi_{1}$ pour différentes valeurs de $\Pi_{3}\left(\Pi_{3}=\Pi_{2} / \Pi_{1}\right.$ de façon à éliminer le paramètre temps).

\subsection{Critères de rupture}

\subsubsection{Critères de rupture en paramètres $\left(P_{e}\right)$}

Les expériences de rupture en laboratoire conduisent directement à l'obtention, par points, de la surface limite tracée dans l'espace des paramètres $\left(\mathrm{P}_{e}\right)$. Cette surface ne dépend d'aucune hypothèse et relève simplement des mesures en laboratoire.

\subsubsection{Critères de rupture en variables locales . (cas monophasique)}

Il est possible de retranscrire ces critères de rupture sous forme de critères relatifs aux champs locaux existant en bout de fissure, dès lors que l'on dispose de normes sur ces champs et que l'on dispose de formules de passage des paramètres $\mathrm{P}_{e}$ à ces normes.

Dans les expériences thermo-hydro-mécaniques, on est actuellement limité comme on vient de le voir précédemment à des interprétations thermo-mécaniques, dans des matériaux condérés comme monophasiques. Sous cette réserve, le critère de rupture local le plus simple consiste à supposer qu'il existe une valeur limite $\left(\mathrm{K}_{1}\right)_{\text {crit }}$, comme dans un problème purement mécanique. Un champ de contraintes thermomécaniques caractérisé par $\mathrm{K}_{\mathrm{t}}(\mathrm{t})$, à l'instant $\mathrm{t}$, sera critique si le critère $\mathrm{K}_{\mathrm{I}}(\mathrm{t})-\mathrm{K}\left(\mathrm{t}_{\mathrm{f}}\right.$ crit $=0$ est satisfait.

\subsubsection{Critères de rupture en paramètres $\left(P_{s}\right)$ - (cas monophasique)}

Dans le cas d'une approche par mise en situation globale, la surface limite, dans l'espace des paramètres $\left(\mathrm{P}_{\mathrm{s}}\right)$ du site, serait identique à la surface limite dans l'espace des paramètres $\left(\mathrm{P}_{\mathrm{e}}\right)$. Elle ne dépendrait d'aucune hypothèse et relèverait directement des résultats expérimentaux au laboratoire.

Dans le cas de la mise en situation locale, au contraire, on ne peut pas s'affranchir des hypothèses relatives au calcul des champs locaux, pour passer de la surface limite en paramètres $\left(\mathrm{P}_{\mathrm{e}}\right)$ à la surface limite en paramètres $\left(\mathrm{P}_{\mathrm{s}}\right)$.

Sous réserve des hypothèses de la thermo-mécanique de la rupture monophasique, on peut écrire cette surface limite sous la forme:

$$
\begin{aligned}
& \frac{2}{\sqrt{\Pi}} \sqrt{a_{s}}\left(p_{i s}-\sigma_{3 s}\right)+g_{s}\left(\frac{t_{s}}{\tau_{s}}, \frac{\chi_{s} t_{s}}{a_{s}^{2}}\right) \\
& \sqrt{a_{s}} \cdot \alpha_{s} \cdot \Delta T_{s}, \lambda_{s}-K_{\text {lent }}=0
\end{aligned}
$$

\section{EXPÉRIENCES ET RÉSULTATS}

\subsection{Dispositif expérimental de rupture thermo-hydro-mécanique}

\subsubsection{Eprouvettes}

Les dimensions des éprouvettes utilisées sont les suivantes:

Rayon du forage $r_{e}=5 \cdot 10^{-3} \mathrm{~m}$

Rayon extérieur de l'éprouvette $R_{e}=1,2 \cdot 10^{-1} \mathrm{~m}$

Hauteur de l'éprouvette $\mathrm{H}_{e}=1,6 \cdot 10^{-1} \mathrm{~m}$

Profondeur des fissures $\mathrm{a}_{e}=5 \cdot 10^{-3} \mathrm{~m}$

Les fissures sont réalisées à l'aide d'un petit disque diamanté situé à l'extrémité d'un bras et entraîné par des courroies crantées reliées à un moteur à vitesse variable (fig. 4).

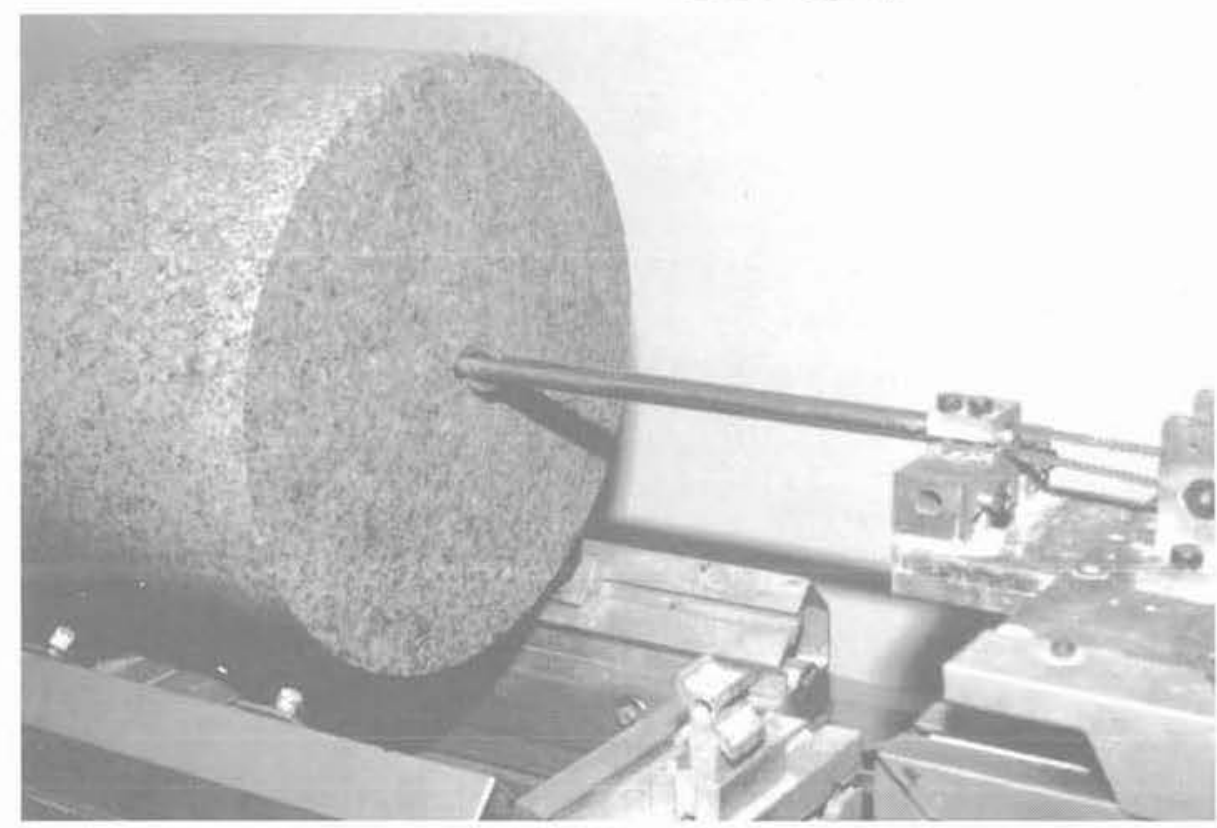

Fig. 4. - Appareillage de création de fissure: détail du bras avec le disque diamanté. Fig. 4. - Apparatus for creating a fracture; detail of arm with diamond cutting disc. 


\subsubsection{Banc d'essais}

Le banc d'essai est constitué d'une cellule et de périphériques permettant d'atteindre des pressions de $20 \mathrm{MPa}$ et des températures de $200{ }^{\circ} \mathrm{C}$.

\section{a. Cellule d'essais (fig. $5 a$ et $5 b$ )}

L'échantillon de roche, emprisonné entre un flasque intérieur et un piston supérieur, est soumis à une pression de confinement $\mathrm{p}_{\mathrm{oe}}$ par l'intermédiaire d'une huile résistant à haute température. Dans le forage central et les fractures, situées au centre de l'échantillon, circule le fluide caloporteur (eau) à une pression $p_{\text {fe }}$ et à une température $T_{f e}$. Des joints toriques en viton assurent l'étanchéité entre les pièces mobiles, mais aussi sur les faces inférieure et supérieure de l'éprouvette, entre les circuits d'huile et d'eau. Le piston exerce sur l'échantillon une contrainte verticale $\sigma_{v e}$ qui engendre des forces de frettage. Il a été nécessaire d'effectuer des essais préliminaires pour évaluer ces forces ainsi que pour déterminer la température en bout de fracture connaissant la température au centre du forage. Cette cellule est placée dans une enceinte de chauffage thermostatée qui permet de maintenir la cellule d'essais, l'échantillon et le fluide caloporteur à la température initiale $\mathrm{T}_{\text {re }}$.

\section{b. Périphériques (fig. 6)}

Outre les périphériques classiques permettant de créer et de réguler les pressions et les températures, il a

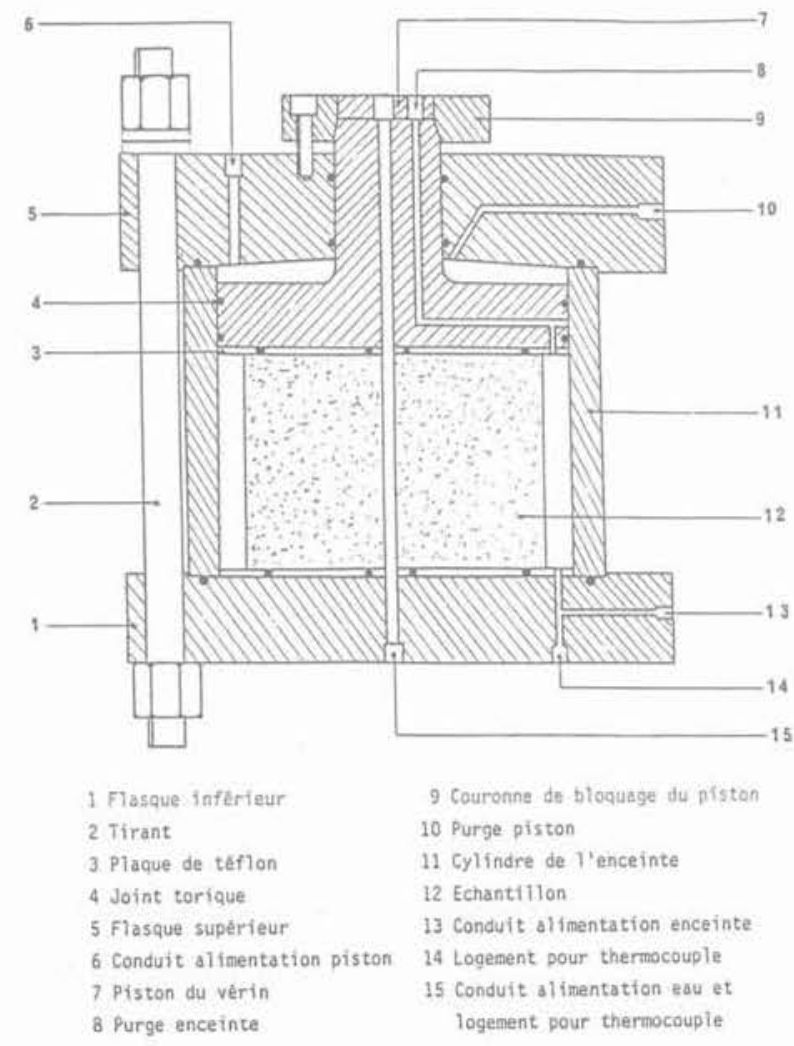

Fig. 5a. - Schéma de la cellule d'essais. Fig. 5a. - Scheme of test cell. fallu mettre au point un périphérique pour maîtriser le refroidissement de l'eau dans la fracture. Ce périphérique comprend deux boucles thermiques de circulation de fluide :

- une boucle fermée, haute température et haute pression, qui véhicule l'eau dans la fracture par l'intermédiaire d'un circulateur hauteur pression entraîné, à l'extérieur de l'enceinte, par un moteur à vitesse variable relié à un joint tournant :

- une boucle ouverte, basse température et basse pression, reliée à un ballon d'eau froide, qui permet, à l'aide d'un échangeur thermique à contre-courant et d'une vanne trois voies motorisée, de refroidir l'eau dans le forage et la fracture.

Ce montage permet d'avoir une certaine souplesse sur la fonction $\mathrm{T}_{f e}\left(\mathrm{t}_{e}\right)$, en partant de la température initiale $T_{\mathrm{fe}}=\mathrm{T}_{\mathrm{re}}$.

\subsubsection{Divers types d'essais}

Les essais ont été effectués sur des échantillons de calcaire - calcaire de Baruthel (Gard) - et sur des

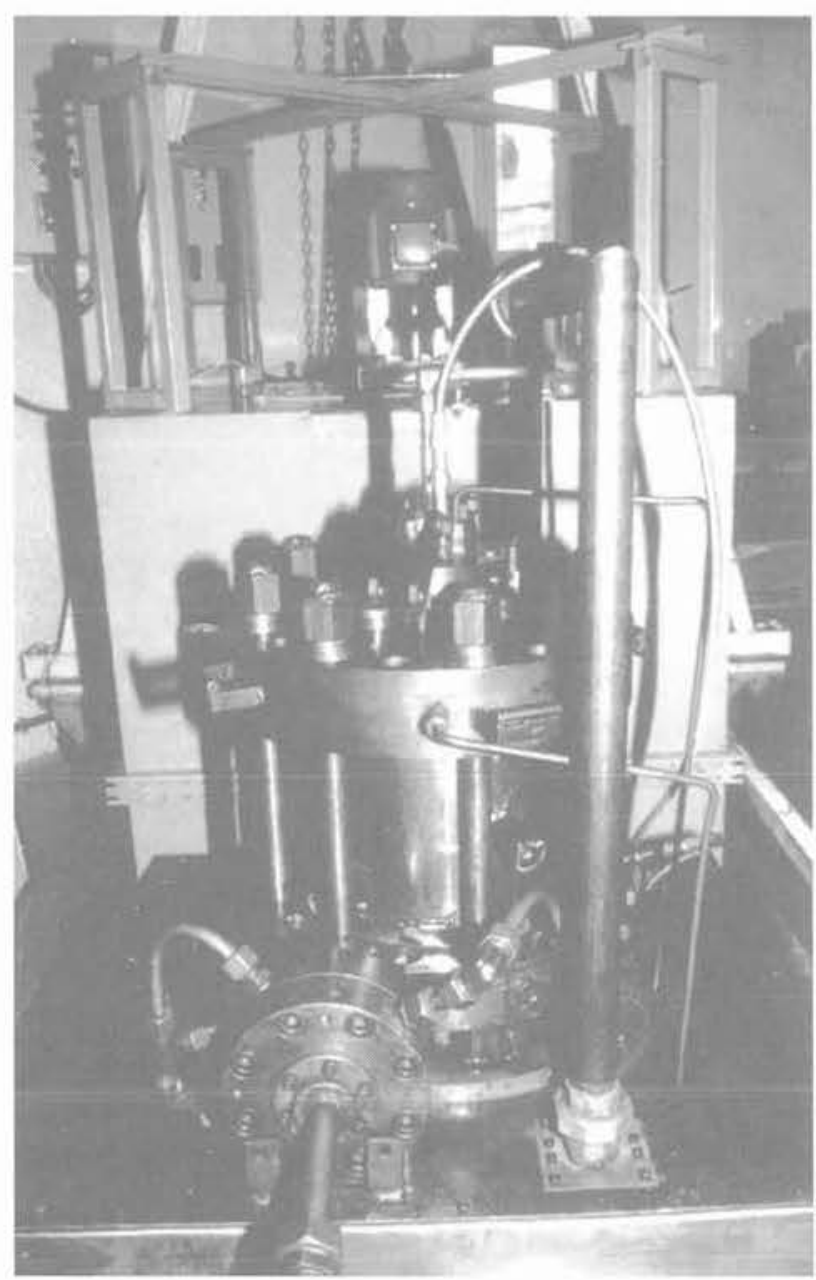

Fig. $5 b$ - Vue d'ensemble de la cellule d'essais, du circulateur haute pression, de l'échangeur et de l'étuve. Fig. $5 b$. - Overall view of test cell, high pressure pump, exchanger and heat chamber. 


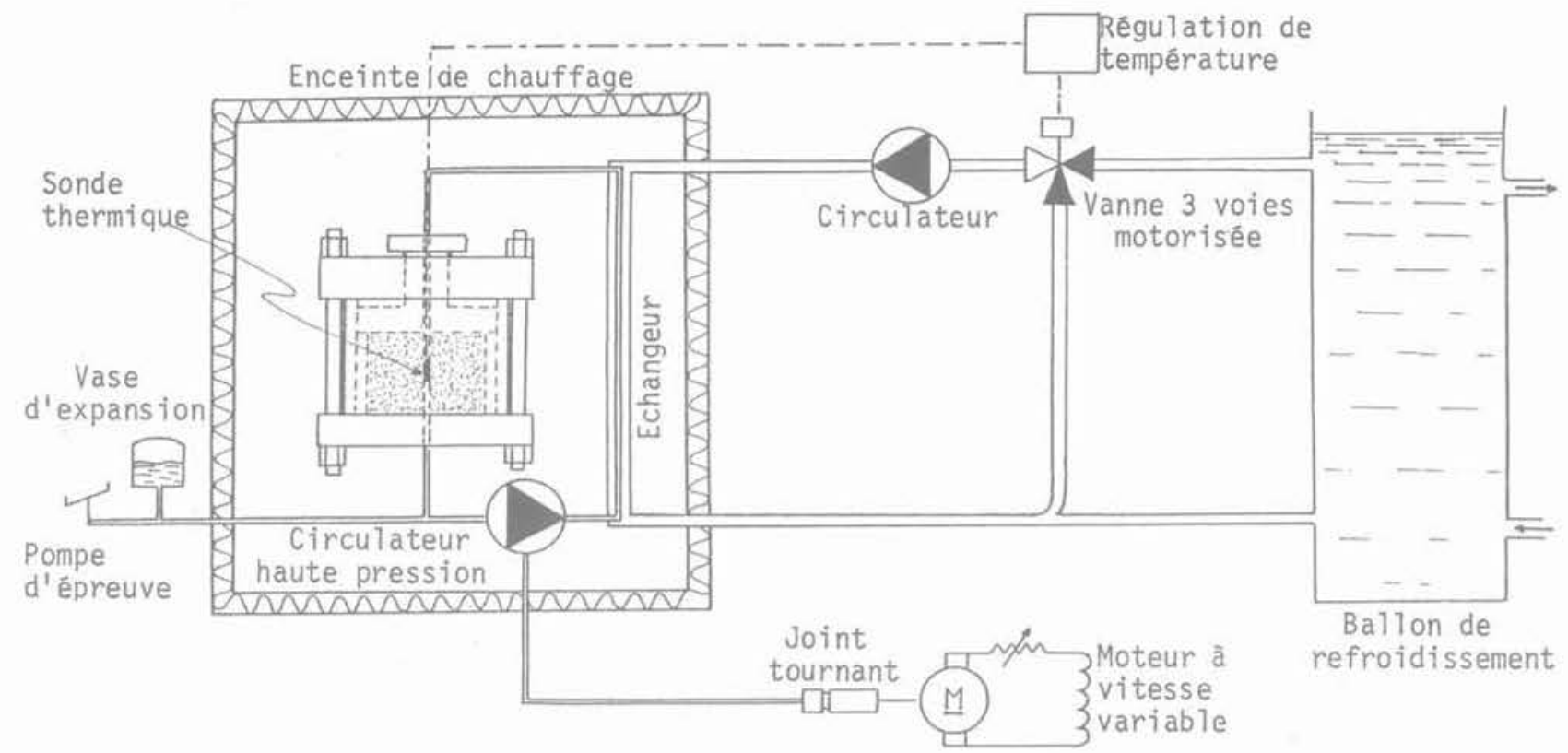

Fig. 6. - Schéma du banc d'essais.

Fig. 6. - Scheme of test apparatus.

échantillons de granite - granite de Lanhelin (Bretagne) - qui peuvent être considérés comme homogènes et isotropes et dont les caractéristiques thermomécaniques ont pour valeurs :

Module d'Young

Coefficient de Poisson

Porosité

Perméabilité à l'eau

Masse volumique

Chaleur massique

Coefficient de dilatation linéaire

Conductivité thermique

Le mode opératoire est représenté sur la figure 7 dans l'espace des trois paramètres de chargement : pression de confinement $\left(\mathrm{p}_{\mathrm{oe}}\right)$, température du fluide $\left(\mathrm{T}_{\mathrm{fe}}\right)$ et pression du fluide $\left(\mathrm{p}_{\mathrm{te}}\right)$.

a. Etablissement des conditions initiales

- Etablissement de l'étreinte latérale poe et verticale $\sigma_{\text {ve }}$ (Cette dernière contrainte doit être suffisante pour éviter la formation d'une fracture perpendiculaire au forage) ;

- mise en pression du forage sous une pression $\left(\mathrm{p}_{\mathrm{oe}}\right)$;

- montée en température de l'ensemble jusqu'à

$\mathrm{T}_{\mathrm{fe}}=\mathrm{T}_{\mathrm{re}}$ compris entre $50^{\circ} \mathrm{C}$ et $200^{\circ} \mathrm{C}$ suivant les expériences.

\section{b. Essai de rupture}

Deux types d'essais de rupture ont été réalisés:

b1. Essais de fracturation hydraulique

A partir des conditions initiales, et en opérant à température constante $\mathrm{T}_{\text {fe }}$, on augmente la pression de
Calcaire

$27,810^{3} \mathrm{MPa}$

0,24

$12-15 \%$

$2,910^{-10} \mathrm{~m} / \mathrm{s}$

$2,305 \mathrm{~g} / \mathrm{cm}^{3}$

$0,88 \mathrm{~J} \cdot \mathrm{g}^{-1} \cdot{ }^{\circ} \mathrm{C}^{-1}$ à $70^{\circ} \mathrm{C}$

$0,96 \mathrm{~J} \cdot \mathrm{g}^{-1} \cdot{ }^{\circ} \mathrm{C}^{-1}$ à $150^{\circ} \mathrm{C}$

$2,35 \mathrm{~W} \cdot \mathrm{m}^{-1} \cdot{ }^{\circ} \mathrm{C}^{-1}$
Granite

$69,910^{3} \mathrm{MPa}$

0,32

$0,7-0,8 \%$

$1,6510^{-14} \mathrm{~m} / \mathrm{s}$

$2,621 \mathrm{~g} / \mathrm{cm}^{3}$

$0,80 \mathrm{~J} \cdot \mathrm{g}^{-1} \cdot{ }^{\circ} \mathrm{C}^{-1}$ à $70{ }^{\circ} \mathrm{C}$

$0.96 \mathrm{J.g}^{-1} \cdot{ }^{\circ} \mathrm{C}^{-1}$ à $150^{\circ} \mathrm{C}$

idem

$3,29 \mathrm{~W} \cdot \mathrm{m}^{-1} \cdot{ }^{\circ} \mathrm{C}^{-1}$

Pression de confinement $\left(p_{0 e}\right)$

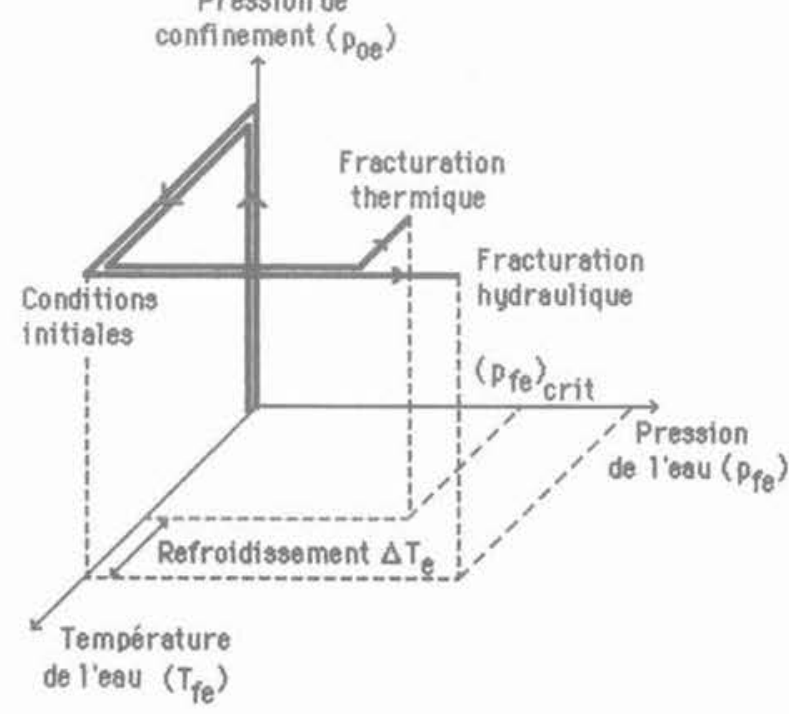

Fig. 7. - Cheminement du mode opératoire. Fig. 7. - Operation sequence. 
l'eau pfe dans la fracture jusqu'à la rupture de la roche. Cet essai sert de référence et permet de déterminer la pression de claquage hydraulique isotherme $\left(p_{\text {fel }}\right)_{\text {crit. }}$

Ces essais de fracturation hydraulique ont été effectués de deux façons, afin de rendre compte de l'influence de la pression interstitielle sur la rupture de la roche:

- essai par a montée rapide " en pression : le termps de montée en pression de l'eau dans la fracture n'excède pas 30 s ;

- essai par « montée en pression par paliers \& : la montée en pression de l'eau dans la fracture s'effectue par paliers d'amplitude 0,5 à $1.0 \mathrm{MPa}$ appliqués pendant $5 \mathrm{mn}$, ordre de grandeur des temps de refroidissement avant rupture dans les essais de fracturation thermique.

\section{b2. Essais de fracturation thermique}

A partir des conditions initiales, sous une pression constante $\mathrm{p}_{\mathrm{fe}}$ égale à un certain pourcentage de la pression $\left(\mathrm{p}_{\mathrm{fe}}\right)_{\text {crit }}$ précédernment déterminée, on refroidit l'eau du forage suivant une loi donnée $T_{f e}=T_{r e}$ $-\Delta \mathrm{T}_{e}$ th $\left(\mathrm{t}_{e} / \tau_{e}\right)$ et l'on note l'instant de la rupture $\mathrm{t}_{e}$. - Les températures initiales $\mathrm{T}_{\text {re }}$ sont de $70^{\circ} \mathrm{C}$, $120^{\circ} \mathrm{C}, 150^{\circ} \mathrm{C}$ et $185^{\circ} \mathrm{C}$;

- les amplitudes maximales de refroidissement $\Delta \mathrm{T}_{e}$ varient entre $10^{\circ} \mathrm{C}$ et $70^{\circ} \mathrm{C}$;

- les temps de relaxation $\tau_{e}$ sont faibles et varient entre $30 \mathrm{~s}$ et $130 \mathrm{~s}$; les refroidissements sont donc rapides et les temps de sollicitation $t_{e}$ inférieurs à 5 minutes.

La rupture est atteinte quand on constate une chute brutale de $p_{\text {fe. }}$.

\subsection{Résultats}

\subsubsection{Essais de fracturation hydraulique}

A. Paramètres de rupture $\left(P_{e}\right)_{\text {eritiques }}$ au laboratoire Les courbes (1) et (2) des figures 8 et 9 représentent, pour le calcaire et pour le granite, la variation de $\left(\Delta \mathrm{p}_{e}\right)_{\text {crit }}=\left(\mathrm{p}_{\mathrm{fe}}\right)_{\mathrm{crit}}-\mathrm{p}_{\mathrm{oe}}$ qui conduit à la rupture par claquage purement hydraulique, et ceci à différentes températures $T_{\text {re }}$ de la roche.

\section{-Importance de l'environnement hydraulique}

On constate que la pression de claquage hydraulique est beaucoup plus importante par « montée rapide » (courbes 1) que par « paliers » (courbes 2), l'eau n'ayant pas eu le temps d'envahir les pores. Ce résultat, bien connu [10, 15], a aussi été mis en évidence lors de l'étude sur la stabilité des parois d'un échangeur géothermique $[3,4,6,8]$. On confirme ainsi, s'il en était besoin, que les conditions de mise en situation au plan hydraulique sont fondamentales.

\section{- Importance de l'environnement thermique}

Pour le calcaire étudié, la pression de claquage hydraulique $\left(\mathrm{p}_{\mathrm{fe}}\right)_{\text {crit }}$ est indépendante de la température de la roche (entre 50 et $200^{\circ} \mathrm{C}$ ), les courbes (1) et (2) étant horizontales.
Pour le granite, $\left(\mathrm{p}_{\mathrm{fe}}\right)_{\text {crit }}$ diminue avec la température de la roche ; la résistance du granite diminue lorsque la température augmente (entre 50 et $200^{\circ} \mathrm{C}$ ).

\section{B. Champs locaux en bout de fissure}

On peut chercher à transformer ces résultats en valeurs de ténacité. Cette transformation peut être considérée comme possible, pour les courbes (1), où le claquage hydraulique est effectué avec une montée rapide en pression de l'eau dans le forage, mais n'est uraiment plus licite dans le cas des courbes (2), où l'eau a eu le temps d'envahir les pores.

A titre d'illustration, les valeurs des ténacités correspondant à ces courbes (1), calculées par l'expression "monophasique " :

$$
\mathrm{K}_{\mathrm{le}}^{\mathrm{FH}}=\sqrt{\Pi\left(\mathrm{r}_{e}+\mathrm{a}_{e}\right)} \cdot\left[\left(\mathrm{p}_{\mathrm{fe}}\right)_{\text {crit }}-\mathrm{p}_{\mathrm{oe}}\right]
$$

sont données sur les courbes (1) des graphiques 10 (a et b), relatifs respectivement au calcaire et au granite.

\section{Paramètres de rupture $\left(P_{s}\right)_{\text {critiques sur le site }}$}

La transposition sur le site peut s'effectuer uniquement dans des circonstances où la roche est supposée non envahie par l'eau. Ceci restreint le champ d'application des résultats de laboratoire, dans l'attente d'une interprétation biphasique.

\subsubsection{Essais de fracturation thermique}

\section{A. Paramètres de rupture $\left(P_{e}\right)_{\text {critques }}$ au laboratoire}

Sur les figures 8 et 9 , la frange hachurée entre les courbes (2) et (3) représente le domaine des $\Delta \mathrm{p}_{e}=$ $p_{f e}-p_{o e}$ pour lesquels on a obtenu fracturation thermique avant 5 minutes de refroidissement, dans les conditions de nos expériences. Pour des valeurs de $\Delta \mathrm{p}_{e}$ situées en dessous de la courbe (3), c'est-àdire pour des pourcentages $\mathrm{p}_{\mathrm{fe}} /\left(\mathrm{p}_{\mathrm{fe}}\right)_{\text {crit }}$ inférieurs à $70 \%$, on n'a pas obtenu de fracturation thermique. Cette frange de $\Delta \mathrm{p}_{e}$, dans laquelle il peut $\mathrm{y}$ avoir rupture de la roche par fracturation thermique, est relativement étroite malgré des amplitudes de refroidissement déjà notables mais pourrait être plus large pour des refroidissements plus importants en forage.

Il convient de bien noter que les températures $T_{\text {re }}$ portées en abscisse représentent les températures de la roche avant tout refroidissement. Cette représentation de l'espace des paramètres $P_{e}$ est imparfaite, et doit être complétée par les paramètres de la loi de refroidissement.

\section{B. Champs locaux en bout de fissure}

La transposition de ces résultats sous forme de champs locaux en bout de fissure se heurte comme précédemment à l'influence des pressions d'eau. En effet, les expériences de refroidissement du fluide en forage ont été effectuées après avoir établi une pression d'eau non négligeable dans la roche. Aucune expérience thermique n'a été faite simultanément avec une montée en pression rapide.

De plus le calcul de facteurs d'intensité de contraintes thermo-mécaniques se heurte à une autre difficulté 


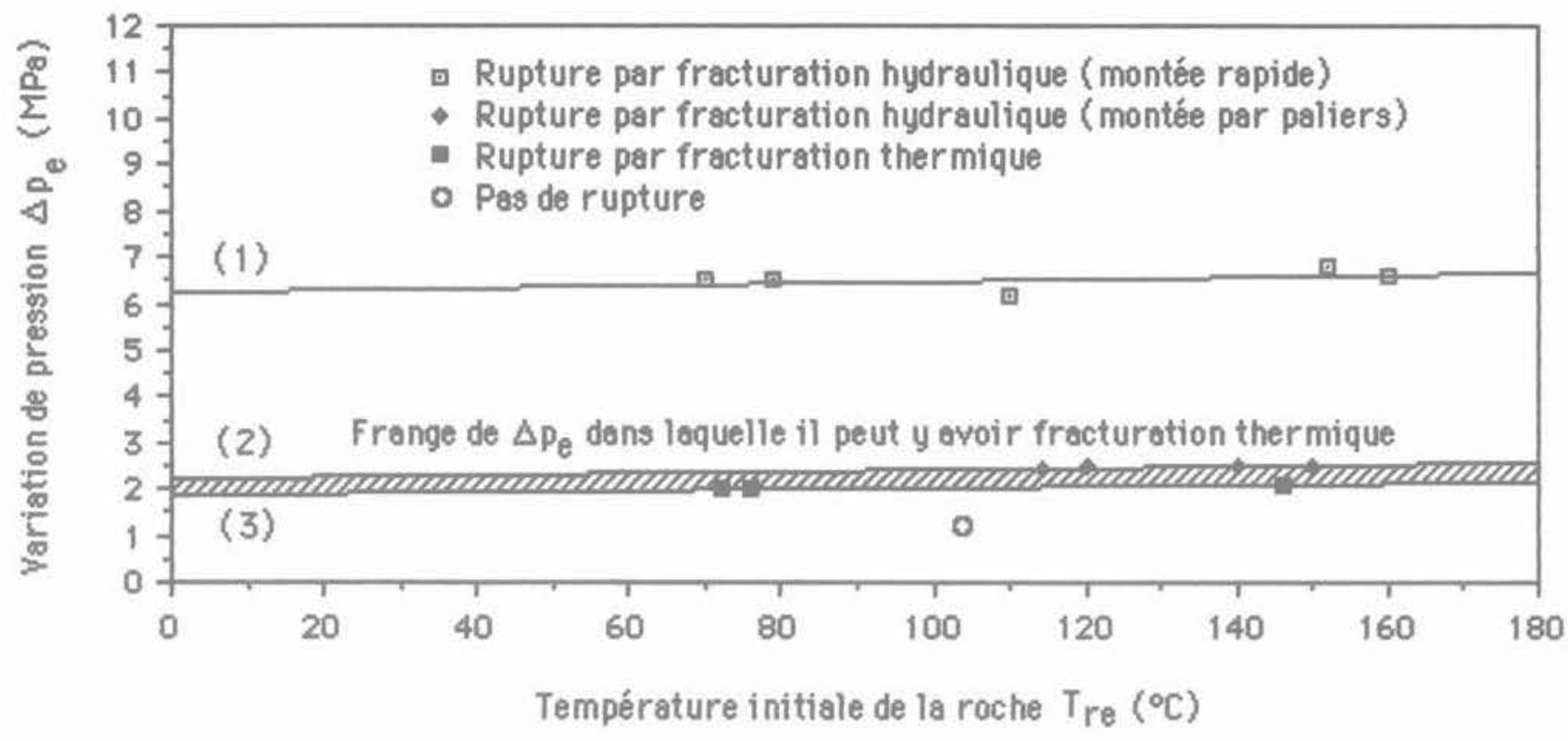

Fig. 8. - Essais sur calcaire. Variation de $\Delta p_{e}$ en fonction de $T_{\text {re. }}$

Fig. 8. - Test on limestone - Variation of $\Delta p_{e}$ as function of $T_{r e}$.

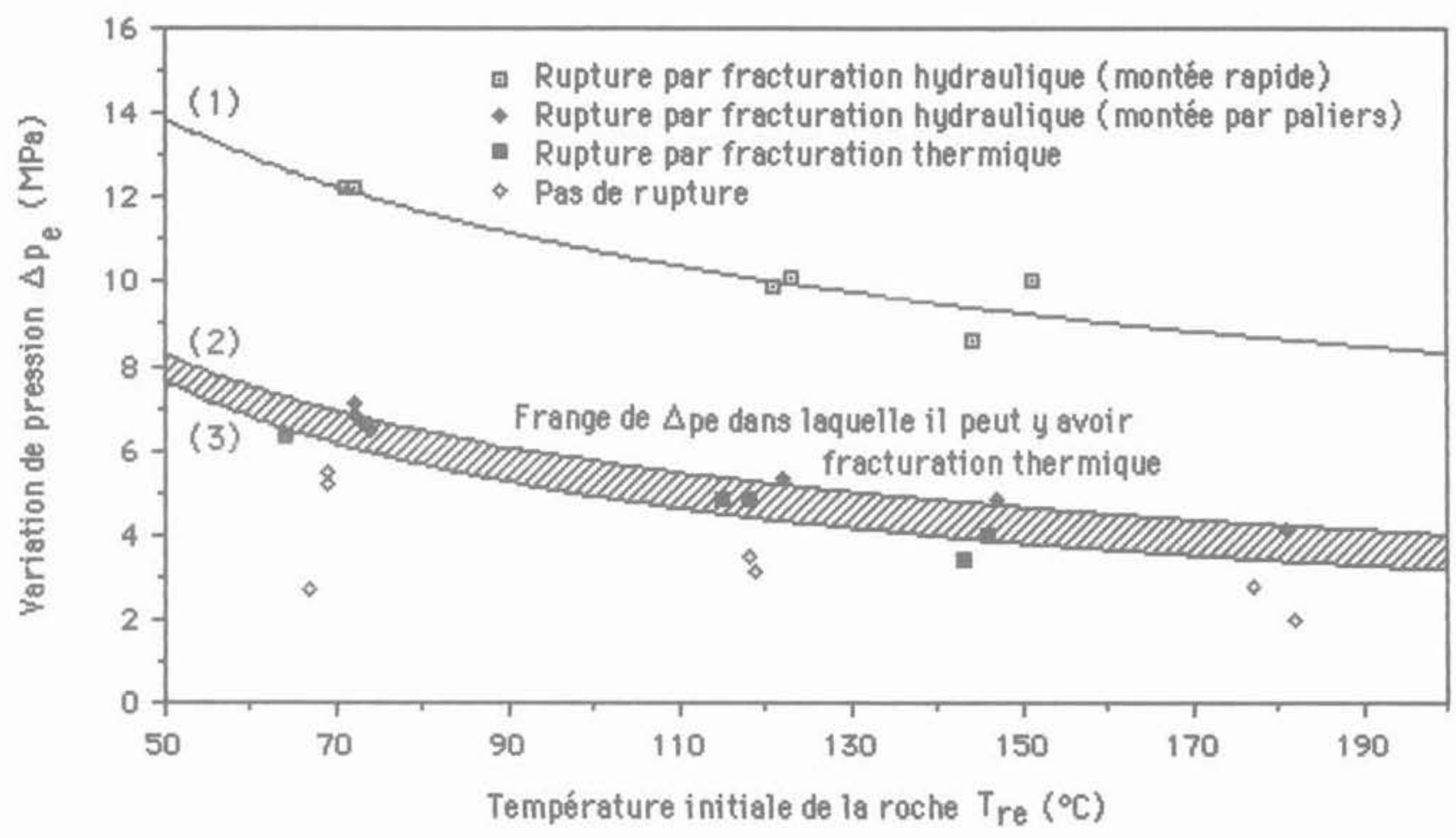

Fig. 9. - Essais sur granite. Variation de $\Delta p_{\varepsilon}$ en fonction de $T_{\text {re. }}$ Fig. 9. - Test on granite - Variation of $\Delta p_{p}$ as function of $T_{r e}$ 

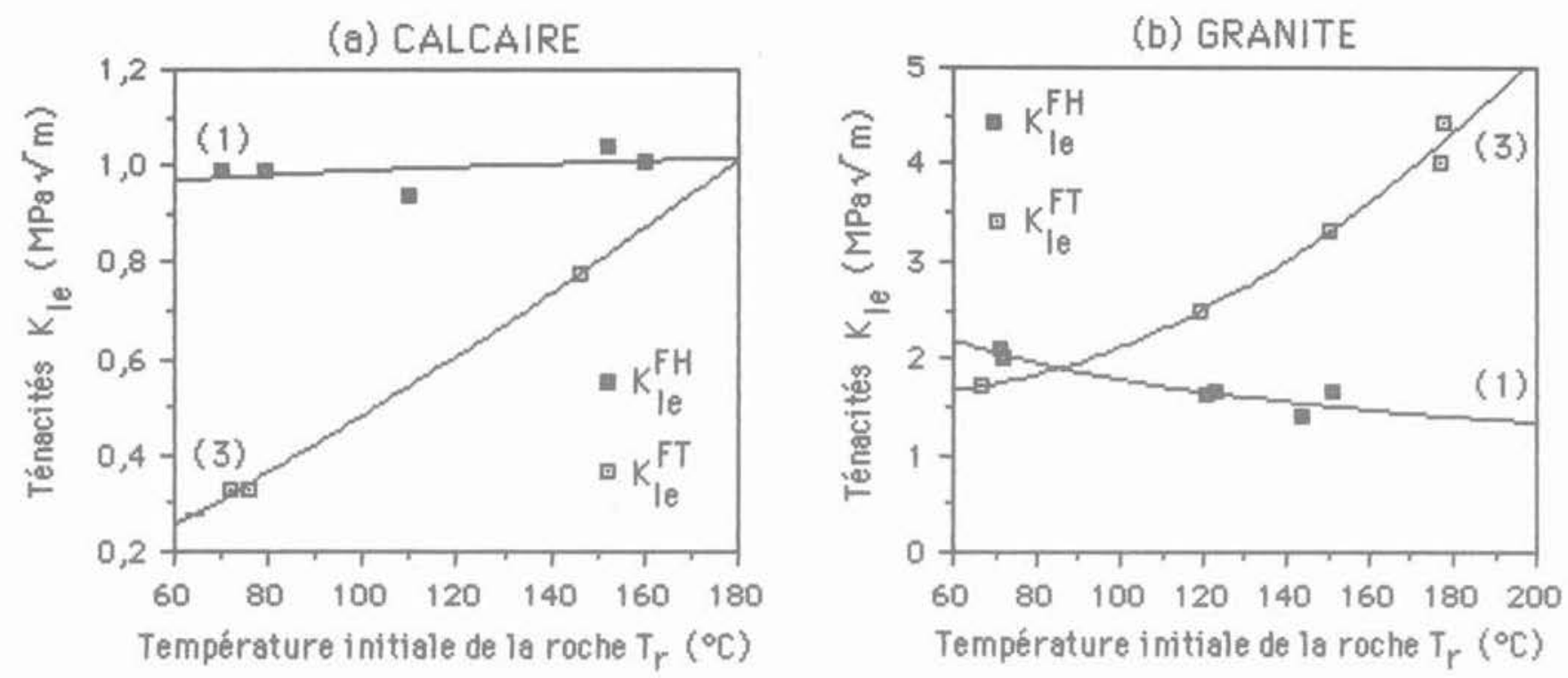

Fig. 10. - Ténacités $K_{l e}$ calculées en monophasique lors d'essais de fracturation hydraulique (FH) et thermique (FT).

Fig. 10 - Tensile strength $K_{\text {le }}$ calculated for monophasic conditions during hydraulic (FH) and thermal (FT) fracturing tests.

qui concerne la valeur à prendre en compte dans la mesure du refroidissement à l'extrémité de fissure. Des mesures très précises ont montré en effet que ces températures variaient très vite à la paroi de la fissure.

Toutes difficultés confondues, on a reporté également sur les graphiques 10 ( $\mathrm{a}$ et b) les valeurs des ténacités obtenues lors de fracturations thermiques (courbes 3 ), calculées par l'expression * monophasique " suivante :

$\mathrm{K}_{\mathrm{le}}^{\mathrm{FT}}=\sqrt{\Pi\left(\mathrm{r}_{e}+\mathrm{a}_{e}\right)} \cdot\left(\mathrm{p}_{\mathrm{fe}}-\mathrm{p}_{\mathrm{e}}\right)$
$+\mathrm{g}_{e}\left(\frac{\mathrm{r}_{e}}{\mathrm{a}_{e}}, \frac{\mathrm{R}_{e}}{\mathrm{a}_{e}}, \frac{\mathrm{t}_{e}}{\tau_{e}} \cdot \frac{\chi_{e} \mathrm{t}_{e}}{\mathrm{a}_{e}{ }^{2}}\right) \sqrt{\mathrm{a}_{e}} \cdot \alpha_{e} \cdot \Delta \mathrm{T}_{e} \cdot \lambda_{e}$

On note un manque évident de cohérence entre les courbes de fracturation hydraulique et de fracturation thermique qui peut s'expliquer, comme indiqué cidessus, par les limites d'une analyse monophasique du phénomène et par la sensibilité à la mesure de lamplitude $\Delta \mathrm{T}_{e}$ du refroidissement.

\section{Paramètres de rupture $\left(P_{s}\right)_{\text {critiques }}$ sur le site}

On conçoit dans ces conditions les difficultés pour parvenir à des enseignements sur les paramètres de rupture in situ. Sous ces réserves, la connaissance des valeurs $\left(\mathrm{K}_{1}\right)_{\text {crit }}$ conduit à l'expression de la surface limite " monophasique * dans l'espace des paramètres $\left(\mathrm{P}_{5}\right)$, comme indiqué au paragraphe 2.3.3.

\section{CONCLUSIONS}

Les expériences de fracturation thermo-hydromécanique de roches préfissurées, conduites tant sur des échantillons de calcaire que de granite, ont con- duit à l'obtention d'une banque importante de résultats de fracturation, qui fait intervenir un grand nombre de paramètres au laboratoire : caractéristiques géométriques, coefficients physiques, conditions thermohydro-mécaniques initiales ou au cours de l'évolution des températures au voisinage de l'extrémité des fissures.

Par rapport à des expériences classiques de fracturation en roches sèches isothermes, l'interprétation se complique pour plusieurs raisons :

- si l'on reste dans le domaine thermo-mécanique pur, quelque peu idéal, des roches sèches, les difficultés d'interprétation ne sont pas théoriques, puisque l'extension à un tel cas de la mécanique de la rupture est acquise. Mais l'interprétation devient délicate, au plan expérimental, vu la difficulté à donner un contenu précis à la mesure des températures au voisinage de l'extrémité de la fracture:

- si l'on aborde le domaine de roches en présence d'eau, en situation hydro-mécanique, alors l'interprétation bute actuellement devant un mur théorique ; - a fortiori il en est de même dans le cas général de sollicitations thermo-hydro-mécaniques.

Dans de telles situations complexes, pour transposer les résultats du laboratoire sur le site, deux voies sont alors envisageables:

- soit la voie directe de lapproche par mise en situation globale, praticable immédiatement, mais seulement dans certaines applications, par exemple pour des problèmes de tenue de forages, où les conditions en laboratoire simulent correctement la situation in situ ;

- soit la voie indirecte de mise en situation locale, dans le cadre de nouveaux développements théoriques en thermo-hydro-mécanique de la rupture, développements qui sont actuellement en cours. 


\section{REMERCIEMENTS}

Cette recherche a fait l'objet de contrats passés avec la Commission des Communautés Européennes que nous tenons à remercier.

Le CNRS a apporté une aide conjointe, dans le cadre de I'UA 04859 .

\section{BIBLIOGRAPHIE}

[1] BARTHELEMY B. (1980), Notions pratiques de mécanique de la rupture. Edit. Eyrolles, Paris.

[2] BATCHELOR A.S. (1983), Hot Dry Rock reservoir stimulation in the UK: an extended summary. 3rd Int. Seminar on the Results of European Communities, Geothermal Energy, Munich (nov. 29 - déc. 1, 1983).

[3] BERTHOMIEU G., JOUANNA P. (1983), Wall stability of a deep geothermal reservoir under thermal actions. 3rd Int. Seminar on the Results of European Communities, Geothermal Energy. Munich (nov. 29 - déc., 1983).

[4] BERTHOMIEU G., JOUANNA P. (1984), Stability of rock faces subjected to temperature change - Application to hot dry granite. Int. J. Rock Mech. Min. Sci \& Geomech., vol. 21, $n^{\circ} 5$, pp. 277-287.

[5] BERTHOMIEU G., CHEISSOUX J.L. DABERT J.L., JOUANNA P. (1984), Stress intensity factor $K$, under variable thermal conditions. 3rd Int. Conf. Num. Meth. in Fracture, Swansea, pp. 481-494.

[6] BERTHOMIEU G., JOUANNA P. (1985), Stabilité d'un échangeur géothermique profond sous sollicitations thermiques. Revue roumaine des sciences techniques, Mécanique appliquée, Tome $30, n^{\circ} 1, p p \cdot 67-74$.

[7] BERTHOMIEU G., JOUANNA P. (1985), Conditions de similitude en thermomécanique de la rupture. $7^{e}$ Congrès Français de Mécanique, Bordeaux (2-6 sept 1985).

[8] BERTHOMIEU G. (1987), Fracturation thermique des roches. Application à la géothermie profonde. Thèse d'Etat, U.S.T.L., Montpellier, 2 juillet 1987.

[9] BUI H.D. (1978), Mécanique de la rupture fragile. Edit Masson, Paris.

[10] CORNET F. (1978), Application de la fracturation hydraulique à la mesure "in situ » des contraintes. Mémoires du B.R.G.M., n 91, Colloque Scientifique J. Goguel, pp. 173-185.

[11] HENRY J.P. (1978), Mécanique linéaire de la rupture appliquée à l'étude de la fissuration et de la fracture de roches calcaires. Thèse d'Etat, U.S.T. Lille.

[12] HOUPERT R., HOMAND-ETIENNE F. (1986). Données récentes sur le comportement des roches en fonction de la température. Ecole d'été de thermomécanique des roches, Alès (8-12 sept, 1986).

[13] SHI G.C. (1973), Handbook of stress intensity factors. Inst. of fracture and solid mechanics, Lehigh University, Pennsylvania.

[14] TAKAHASHI H., SHOJI T, ABE H. (1986), Recent progress and future of \& $G$ project » at Tohoku Univ., Japan. Rapport interne C.C.E.

[15] ZOBACK M.D., RUMMEL F., JUNG R., RALEIGH C.B. (1977), Laboratory hydraulic fracturing experiments in intact and prefractured rock. Int. J. Rock Mech. Min. Sci. \& Geomech., vol. 14 , pp. 49-58. 